\title{
Content-Aware Resource Allocation and Packet Scheduling for Video Transmission Over
} Wireless Networks

\author{
Peshala Pahalawatta, Student Member, IEEE, Randall Berry, Member, IEEE, \\ Thrasyvoulos Pappas, Fellow, IEEE, Aggelos Katsaggelos, Fellow, IEEE
}

\begin{abstract}
A cross-layer packet scheduling scheme that streams pre-encoded video over wireless downlink packet access networks to multiple users is presented. The scheme can be used with the emerging wireless standards such as HSDPA and IEEE 802.16. A gradient based scheduling scheme is used in which user data rates are dynamically adjusted based on channel quality as well as the gradients of a utility function. The user utilities are designed as a function of the distortion of the received video. This enables distortion-aware packet scheduling both within and across multiple users. The utility takes into account decoder error concealment, an important component in deciding the received quality of the video. We consider both simple and complex error concealment techniques. Simulation results show that the gradient based scheduling framework combined with
\end{abstract}

This work was supported by the Motorola Center for Seamless Communication at Northwestern University.

The authors are with the Electrical Engineering and Computer Science Department at Northwestern University. the content-aware utility functions provides a viable method for downlink packet scheduling as it can significantly outperform current content-independent techniques. Further tests determine the sensitivity of the system to the initial video encoding schemes, as well as to non-real-time packet ordering techniques.

Index Terms-Wireless packet Scheduling, video streaming, H.264, HSDPA, cross-layer design

\section{INTRODUCTION}

Since the introduction of the first GSM networks, interest in high speed wireless data transmission has grown rapidly. The demand for higher data rates stems mainly from the need to stream high quality multimedia content to mobile users. Multimedia content, and specifically, streaming video, requires per-user data rates of a few hundred kilobits per second in order to be of useful quality. Recent 
cellular standards such as HSDPA (High Speed Downlink Packet Access) [1], and IEEE 802.16 $(W i M A X)$ [2], aim to provide data rates that enable multimedia communication over wireless networks.

Many proposed cross-layer scheduling and resource allocation methods exploit the timevarying nature of the wireless channel to maximize the throughput of the network while maintaining fairness across multiple users [3][7]. These methods rely on the multi-user diversity gain achieved by selectively allocating a majority of the available resources to users with good channel quality who can support higher data rates [8]-[10]. Many of these methods, such as the proportional fair rule for CDMA 1xEVDO, can be viewed as gradient-based scheduling policies [11]. In these policies, during each time-slot, the transmitter maximizes the weighted sum of each user's rate, where the (time-varying) weights are given by the gradient of a specified utility function. One attractive feature of such policies is that they require only myopic decisions, and hence presume no knowledge of long-term channel or traffic distributions. We focus on networks where a combination of TDM and CDMA or OFDMA can be used to transmit data to multiple users simultaneously. We consider "per-user" system constraints which can depend on the capabilities of the mobile client devices. For such networks, [12] discusses the implementation of gradient-based scheduling schemes.

In [12], the optimization over the available resources is performed at each time-slot while taking into account the fading state of each user, at that time. The utility function used in [12] is defined as either a function of each user's current average throughput, or of each user's queue length or delay of the head-ofline packet. A queue-length based utility can be employed for video streaming applications where the delay constraints are stringent. Such a utility does not, however, take into account the content of each video packet. In multimedia applications, the content of a packet is critical in determining the packet's importance. In this work, we propose a content-aware utility function, which is even better suited for video streaming applications, and compare its performance to that of content-independent schemes.

A wealth of work exists on video streaming in general, and on video streaming over wireless networks, in particular. One area, which has received significant attention has been that of optimal real-time video encoding, where the source content and channel model are jointly considered in determining the optimal source encoding modes [13]-[18]. A thorough review of the existing approaches to joint source channel coding for video streaming can be found in [19]. We, however, focus on downlink video 
streaming where the media server is at a different location from the wireless base station, and the video encoding cannot be adapted to changes in the channel. Therefore, we assume the video is pre-encoded and packetized at the server. Packet scheduling for the streaming of pre-encoded video is also a well-studied topic [20]-[22], where the focus has been on generating resource-distortion optimized strategies for transmission and retransmission of a preencoded sequence of video packets under lossy network conditions. The above methods, however, consider point-to-point streaming systems where a video sequence is streamed to a single client.

Packet scheduling for video streaming over wireless networks to multiple clients has conventionally focused on satisfying the delay constraint requirements inherent to the system. Examples of such work are [23], [24] and [25]. In these methods, the quality of service of the received video is measured only in terms of the packet delay, or packet loss rate. Methods that do consider the media content can be found in [26]-[28]. In [26], a heuristic approach is used to determine the importance of frames across users based on the frame types (I, P, or B), or their positions in a group of pictures. In [27], a concept of incrementally additive distortion among video packets, introduced in [20], is used to determine the importance of video packets for each user. Scheduling across users, however, is performed using conventional, content-independent techniques. In [28], the priority across users is determined as a combination of a content-aware importance measure similar to that in [27], and the delay of the Head Of Line (HOL) packet for each user. At each time slot, all the resources are dedicated to the user with the highest priority. In the model considered here, per user resource constraints or lack of available data, make it advantageous to transmit to multiple users at the same time.

Our main contribution is to propose a distortion aware scheduling scheme for packetbased video transmission over wireless networks where a combination of TDM and CDMA is used. The resource allocation scheme departs from the schemes discussed above in that it is performed at each transmission time slot based only on the instantaneous channel fading states of each user. We consider error robust data packetization at the encoder and realistic error concealment schemes at the decoder. We focus on the gradient-based scheduling scheme proposed in [12] and introduce a content-based utility function that enables optimizing over the actual quality of the received video. Our method orders the encoded video packets by their relative contribution to the final quality of the video, and assigns a utility for 


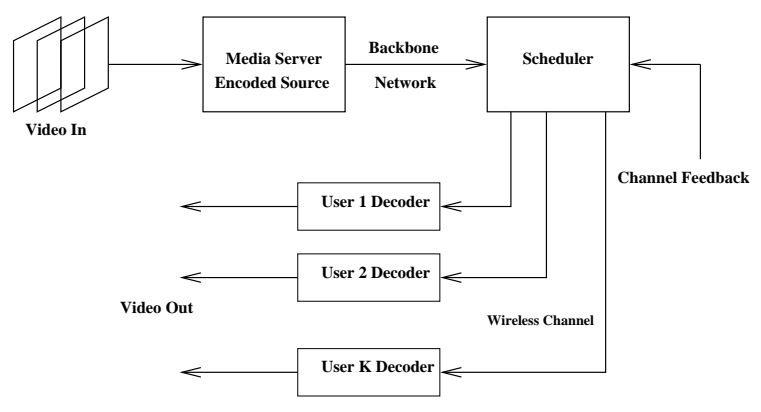

Fig. 1. Overview of multiuser downlink video streaming system

each packet, which can then be used by the gradient-based scheduling scheme to allocate resources across users.

In Sec. II, we give a general overview of the system and also provide some background on video packetization. In Sec. III, we present our main contribution, which is the distortionbased utility function. In Sec. IV, we discuss the resources and constraints inherent to the system and define the general gradient based scheduling problem. A solution is summarized in Sec. V. In Sec. VI, we investigate the performance of the scheduling scheme using both simple and complex error concealment schemes. We also discuss the sensitivity of the scheme to offline packet ordering schemes, and to different video compression schemes. Some final conclusions and avenues for future work are presented in Sec. VII.

\section{SySTEM OVERVIEW}

Figure 1 provides an overview of the system discussed in this paper. We begin with a media server containing multiple video sequences. We assume that each sequence is packetized into multiple data units. Each data unit/packet is independently decodable and represents a slice of the video. In recent video coding standards, such as H.264, a slice could either be as small as a group of a few macroblocks (MBs), or as large as an entire video frame. Each slice header acts as a resynchronization marker, which allows the slices to be independently decodable, and to be transported out of order, and still be decoded correctly at the decoder. Note that, although in terms of decoder operation, each slice is independently decodable, in reality, most frames of a compressed sequence are inter frames, in which MBs may be dependent on macroblocks of previous frames through motion prediction.

Once a video stream is requested by a client, the packets are transmitted over a backbone network to the scheduler at a base station servicing multiple clients. We assume that the backbone network is lossless and of high bandwidth. For simplicity, we assume that all users being served are video users. The scheduling rule can easily accommodate other traffic by assigning them different utility functions. The 
scheduler uses three features of each packet, in addition to Channel State Information (CSI) available through channel feedback, to allocate resources across users. They are, for each packet $m$ of each client $i$, the utility gained due to transmitting the packet (described later), the size of the packet in bits, $b_{i, m}$, and the decoding deadline for the packet, $\tau_{i, m}$. The decoding deadline, $\tau_{i, m}$, stems from the video streaming requirement that all the packets needed to decode a frame of the video sequence must be received at the decoder buffer prior to the playback time of that frame. We can assume that multiple packets (e.g., all the packets in one frame) have the same decoding deadlines.

Any packet left in the transmission queue after its decoding deadline has expired must be dropped since it has lost its value to the decoder. Assuming real-time transmission, the number of transmission time slots available per each video frame can be calculated from the playback time for a frame ( $33 \mathrm{msec}$ for $30 \mathrm{fps}$ video), and the length of each time-slot (e.g., $2 \mathrm{msec}$ for HSDPA). Note that, unlike video conferencing systems, video streaming applications can afford some buffer time at the decoder before starting to play back the video sequence. This is important because, in a compressed sequence, the quality of the first frame, which is intra coded, can have a significant impact on the quality of the following inter coded frames of the same sequence.

The next step in Fig. 1 is that of receiving and decoding the video. At this point, errors in the decoded image are introduced due to the loss of packets in the wireless channel, or due to the dropping of packets from the transmission queue. These errors are typically concealed using an error concealment technique. In general, error concealment techniques use spatial and temporal correlations in the video data so that pixels represented by lost slices are estimated using data from the received slices of the current frame, or a previous frame. Therefore, error concealment introduces an additional dependency between the slices of the sequence.

\section{Content-Aware Utility Function}

The main contribution of our work is to propose a utility function for video streaming that accounts for the dependencies between video packets and the effect that each video packet has on the final quality of the received video. The utility function we propose is especially relevant since it can be used in conjunction with the gradient-based scheduling scheme of [12] to enable content-aware resource allocation across multiple users. In gradient-based scheduling algorithms, packets with a larger first-order change in utility are given priority. The key idea in the proposed method is to sort 
the packets in the transmission buffer for each user based on the contribution of each packet to the overall video quality, and then to construct a utility function such that its gradient reflects the contribution of each packet. A description of the process used to generate packet utilities is given below.

At a given transmission time slot, $t$, for each user, $i$, we pick a group of $M_{i}$ available packets such that each packet $m$ in $M_{i}$ has a decoding deadline, $\tau_{i, m}$, greater than $t$. An obvious approach would be to pick the group of packets with the same decoding deadline that compose the current frame, or group of frames, to be transmitted. We know that each packet $m$ consists of $b_{i, m}$ bits. Note that we are omitting the time index, $t$, for simplicity, since it remains the same throughout this discussion. Now, let $\Pi_{i}=\left\{\pi_{i, 1}, \pi_{i, 2}, \ldots, \pi_{i, M_{i}}\right\}$ be the re-ordered set of packets in the transmission queue such that $\pi_{i, 1}$ will be the first packet of the group to be transmitted. Let $D_{i}\left[\left\{\pi_{i, 1}, \pi_{i, 2}, \ldots, \pi_{i, k_{i}}\right\}\right]$ denote the distortion given that the first $k_{i}$ packets in the queue are transmitted to user $i$ and the remaining $\left(M_{i}-k_{i}\right)$ packets are dropped prior to transmission. Then, we define the user utility for user $i$ after $k_{i}$ packet transmissions as,

$$
U_{i}\left[k_{i}\right]=\left(D_{i}\left[\Pi_{i}\right]-D_{i}\left[\left\{\pi_{i, 1}, \pi_{i, 2}, \ldots, \pi_{i, k_{i}}\right\}\right]\right),
$$

where $D_{i}\left[\Pi_{i}\right]$ is the minimum distortion for the frame that occurs when all packets in the group are received. Note that a new utility function will need to be calculated after these $M_{i}$ packets are sent. The proposed scheme does not depend on the metric used to calculate the distortion. In our numerical work, we define the distortion to be the sum absolute pixel difference between the decoded and error-free frames. For ease of notation, let $\Pi_{i}\left(k_{i}\right)=$ $\left\{\pi_{i, 1}, \ldots, \pi_{i, k_{i}}\right\}$. Then, assuming a simple error concealment scheme (as described in Sec. VIA), we can guarantee that the user utility function is concave and increasing by iteratively choosing each additional packet $\pi_{i, k_{i}+1}$ such that the utility gradient is maximized, i.e.,

$$
\pi_{i, k_{i}+1}=\arg \max _{m \notin \Pi_{i}\left(k_{i}\right)} u_{i, m}\left[k_{i}\right]
$$

where,

$u_{i, m}\left[k_{i}\right]=\frac{D_{i}\left[\Pi_{i}\left(k_{i}\right)\right]-D_{i}\left[\left\{\Pi_{i}\left(k_{i}\right), m\right\} \mid \Pi_{i}\left(k_{i}\right)\right]}{b_{i, m}}$.

In (3), $D_{i}\left[\left\{\Pi_{i}\left(k_{i}\right), m\right\} \mid \Pi_{i}\left(k_{i}\right)\right]$ indicates that the distortion after adding packet $m$ may be dependent on the currently ordered set of packets $\Pi_{i}\left(k_{i}\right)$ from the same group. This will be true if a complex error concealment technique is used at the decoder (See Sec. VI-A.2).

We use the utility gradients, $u_{i, \pi_{k_{i}+1}}\left[k_{i}\right]$ in the gradient based scheduling framework in Sec. IV-C to ensure that the resource allocation will explicitly consider the improvements in video quality for each user. 


\section{Problem Formulation}

\section{A. Channel Resources and Constraints}

We consider a scheme where a combination of TDM and CDMA is used, in which at a given transmission opportunity, $t$, the scheduler can decide on the number of spreading codes, $n_{i}$, (assumed to be orthogonal) that can be used to transmit to a given user, $i$. Note that $n_{i}=0$ implies that user $i$ is not scheduled for transmission at that time slot ${ }^{1}$ (as in the previous section, the time-slot index remains the same throughout this section and is omitted for simplicity). The maximum number of spreading codes that can be handled by each user is determined by the user's mobile device. However, the total number of spreading codes, $N$, that can be allocated to all users, is limited by the specific standard ( $N=15$ for HSDPA). In addition to the number of spreading codes, the scheduler can also decide on the power level, $p_{i}$, used to transmit to a given user. The total power, $P$, that can be used by the base station is also limited in order to restrict the possibility of interference across neighboring cells. Assuming $K$ total users, these constraints

\footnotetext{
${ }^{1}$ In the case of other standards such as CDMA 1xEVDO, only one user can be assigned per time slot. It must be noted that the packet prioritization scheme discussed in Sec. III is applicable to that case, as well.
}

can be written as:

$$
\sum_{i=1}^{K} n_{i} \leq N, \quad \sum_{i=1}^{K} p_{i} \leq P, \quad \text { and }, n_{i} \leq N_{i}
$$

where $N_{i}$ is the maximum number of spreading codes for user $i$.

Our basic assumption in this work is that the constraints of the system will be such that the transmitter may not be able to transmit all the available video packets in the transmission queue of each user in time to meet their decoding deadlines.

\section{B. General Problem Definition}

We assume that the channel state for user $i$, denoted by $e_{i}$, at a given time slot is known based on channel quality feedback available in the system. The value of $e_{i}$ represents the normalized Signal to Interference Noise Ratio (SINR) per unit power and can vary quite rapidly, and in a large dynamic range, over time. Therefore, we assume that $e_{i}$ will be a different but known value at each time slot. Defining $S I N R_{i}=\frac{p_{i}}{n_{i}} e_{i}$ to be the SINR per code for user $i$ at a given time, we can assume that the achievable rate for user $i, r_{i}$, satisfies:

$$
\frac{r_{i}}{n_{i}}=\Gamma\left(\zeta_{i} S I N R_{i}\right),
$$

where $\Gamma(x)=B \log (1+x)$ represents the Shannon capacity for an AWGN channel, where $B$ is the symbol rate per code. Here, $\zeta_{i} \in(0,1]$ represents a scaling factor and determines the 
gap from capacity for a realistic system. This is a reasonable model for systems that use coding techniques, such as turbo codes, that approach Shannon capacity. Setting $\breve{e_{i}}=e_{i} \zeta_{i}$, we can specify the achievable rates for each user as a function of the control parameters $n_{i}$ and $p_{i}$ as follows:

$$
r_{i}=n_{i} B \log \left(1+\frac{p_{i} \breve{e_{i}}}{n_{i}}\right)
$$

Now the resource allocation problem becomes one of specifying the $n_{i}$ and $p_{i}$ allocated to each user such that a target rate, $r_{i}$, can be achieved. In the following, we assume that the channel quality feedback and the modulation and coding schemes are sufficiently good to avoid losses due to fading. In HSDPA, hybrid ARQ can also be used to recover from losses.

\section{Gradient-Based Scheduling Framework}

The key idea in the gradient-based scheduling technique is to maximize the projection of the achievable rate vector, $\mathbf{r}=\left(r_{1}, r_{2}, \ldots, r_{K}\right)$ on to the gradient of a system utility function [12]. The system utility function is defined as:

$$
\mathbf{U}_{i}=\sum_{i=1}^{K} U_{i}
$$

where $U_{i}$ is a concave utility function. In a content-independent scheme, $U_{i}$ can be a function of the average throughput for user $i$, or the delay of the head-of-line packet. In the proposed content-aware scheme, we define $U_{i}$ to be a function of the decoded video quality as in (1). Now, the gradient based resource allocation problem can be written as:

$$
\max _{\mathbf{r} \in \mathcal{C}(\breve{\mathbf{e}}, \chi)} \sum_{i=1}^{K} w_{i} u_{i, \pi_{i, k_{i+1}}}\left[k_{i}\right] r_{i}
$$

where, as in (3), $k_{i}$ denotes the number of packets already transmitted to user $i$, and $\pi_{i, k_{i+1}}$ denotes the next packet in the ordered transmission queue. The constraint set, $\mathcal{C}(\breve{\mathbf{e}}, \chi)$, denotes all the achievable rates given ĕ, the vector containing the instantaneous channel states of each user, and $\chi$ the set of allowable $\mathbf{n}=\left(n_{1}, n_{2}, \ldots, n_{K}\right)$ and $\mathbf{p}=\left(p_{1}, p_{2}, \ldots, p_{K}\right)$, the vectors containing the assigned number of spreading codes, and assigned power levels, of each user, respectively. Here, $w_{i}$ indicates an additional weighting used to attain fairness across users over time. In our numerical work, we have considered a content-based technique for determining $w_{i}$ based on the distortion in user $i$ 's decoded video given the previously transmitted set of packets (i.e., user's with poor decoded quality based on the previous transmissions will be assigned larger weights in order to ensure fairness over time). (8) maximizes a weighted sum of the rates assigned to each user where the weights correspond to the gradients of the specified utility function. After each time-slot, the weights will be re-adjusted based on the packets scheduled in the previous slot. The constraint set will also change due to 
changes in the channel states.

Now, taking into account the system constraints specified in (4), as well as the formula for calculating each user's achievable rate specified in (6), we can formulate the optimization problem as:

$$
V^{*}:=\max _{(\mathbf{n}, \mathbf{p}) \in \chi} V(\mathbf{n}, \mathbf{p})
$$

subject to:

$$
\sum_{i=1}^{K} n_{i} \leq N, \quad \sum_{i=1}^{K} p_{i} \leq P
$$

where:

$$
V(\mathbf{n}, \mathbf{p}):=\sum_{i=1}^{K} w_{i} u_{i, \pi_{i, k_{i}+1}} n_{i} \log \left(1+\frac{p_{i} \breve{e_{i}}}{n_{i}}\right)
$$

and,

$$
\chi:=\left\{(\mathbf{n}, \mathbf{p}) \geq 0: n_{i} \leq N_{i} \forall i\right\} .
$$

\section{Additional Constraints}

In addition to the main constraints specified above, a practical system is also limited by some "per-user" constraints. Among them are, a peak power constraint per user, a maximum SINR per code constraint for each user, and a maximum and minimum rate constraint determined by the maximum and minimum coding rates allowed by the coding scheme.

All of the above constraints can be grouped into a per user power constraint based on the SINR per code for each user [12]. This constraint can be viewed as:

$$
S I N R_{i}=\frac{p_{i} \breve{e_{i}}}{n_{i}} \in\left[\breve{s}_{i}\left(n_{i}\right), s_{i}\left(n_{i}\right)\right], \quad \forall i,
$$

where $\breve{s}_{i}\left(n_{i}\right) \geq 0$. For the purposes of this work, we consider cases where the maximum and minimum SINR constraints are not functions of $n_{i}$, i.e, $S I N R_{i} \in\left[\breve{s}_{i}, s_{i}\right]$, as with a

maximum SINR per code constraint. In this case, the constraint set in (11) becomes,

$$
\chi:=\left\{(\mathbf{n}, \mathbf{p}) \geq 0: n_{i} \leq N_{i}, \breve{s}_{i} \leq \frac{p_{i} \breve{e_{i}}}{n_{i}} \leq s_{i} \forall i\right\} .
$$

\section{E. Extension to OFDMA}

Although the above formulation is primariliy designed for CDMA systems, it can also be adapted for use in OFDMA systems under suitable conditions. For example, a common approach followed in OFDMA systems, is to form multiple subchannels consisting of sets of OFDM tones. In the case that the OFDM tones are interleaved to form the subchannels (i.e., interleaved channelization is used), which is the default case, referred to as PUSC (Partially Used SubCarrier), in IEEE 802.16d/e [2], we can assume that the SINR is essentially uniform across all the subchannels for each user. Then, the number of subchannels plays an equivalent role to the number of codes $(N)$ in the CDMA based formulation above. Further details on gradient based scheduling approaches with OFDMA can be found in [29]. 


\section{SOLution}

A solution to the optimization problem of the type given in (9) for the case when the maximum and minimum SINR constraints are not functions of $n_{i}$ is derived in detail in [12]. In this section, we will simply summarize the basic form of the solution.

The Lagrangian for the primal problem in (9) can be defined as:

$$
\begin{aligned}
& L(\mathbf{p}, \mathbf{n}, \lambda, \mu)= \\
& \quad \sum_{i} w_{i} u_{i} n_{i} \log \left(1+\frac{p_{i} \breve{e}_{i}}{n_{i}}\right)+ \\
& \quad \lambda\left(P-\sum_{i} p_{i}\right)+\mu\left(N-\sum_{i} n_{i}\right)
\end{aligned}
$$

Based on this we can define the dual function,

$$
L(\lambda, \mu)=\max _{(\mathbf{n}, \mathbf{p}) \in \chi} L(\mathbf{p}, \mathbf{n}, \lambda, \mu),
$$

which can be analytically computed by first keeping $\mathbf{n}, \lambda, \mu$ fixed and optimizing (14) over $\mathbf{p}$, and then optimizing over $\mathbf{n}$.

The corresponding dual problem is given by,

$$
L^{*}=\min _{(\lambda, \mu) \geq 0} L(\lambda, \mu) .
$$

Based on the concavity of $V$ in (9), and the convexity of the domain of optimization, it can be shown that a solution to the dual problem exists, and that there is no duality gap, i.e., $V^{*}=L^{*}$.

In [12], an algorithm is given for solving the dual problem based on first optimizing over $\mu$ for a fixed $\lambda$ to find,

$$
L(\lambda)=\max _{\mu \geq 0} L(\lambda, \mu),
$$

and then minimizing $L(\lambda)$ over $\lambda \geq 0$. For the first step, $L(\lambda)$ can be analytically computed. The function $L(\lambda)$ can be shown to be a convex function of $\lambda$, which can then be minimized via a one-dimensional search with geometric convergence.

\section{Simulation StUdy}

\section{A. Error Concealment}

\section{1) Simulation Results Using Simple Error}

Concealment: We categorize as simple, any error concealment technique, in which data from packets within the same group, $\Pi_{i}$, are not used for concealment of other lost packets within that group. For example, if each group consists of packets from one video frame, then replacing the pixel values of MBs contained on a lost packet with pixel values from the same location in the previous frame is a commonly used simple error concealment technique. With such techniques, it can be seen that the packet ordering scheme proposed in Sec. III will always provide the best possible ordering of packets within a packet group, such that given only $k_{i}$ out of the total $M_{i}$ packets are actually transmitted, $\Pi_{i}\left(k_{i}\right)$ would be the set of packets that would lead to the highest decoded video quality. 


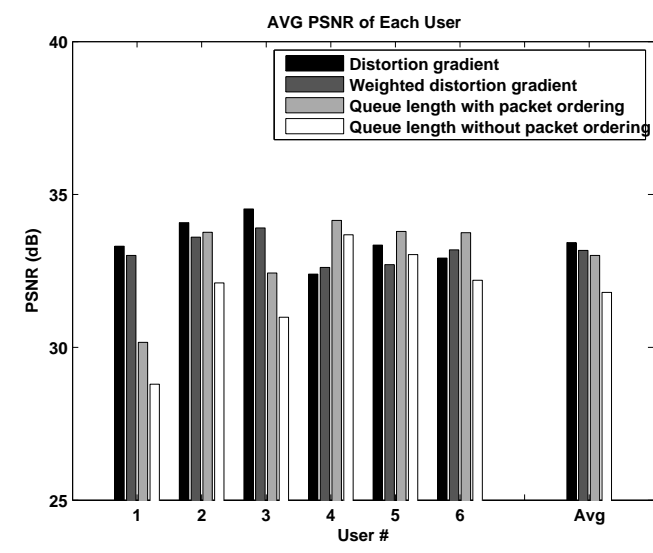

(a)

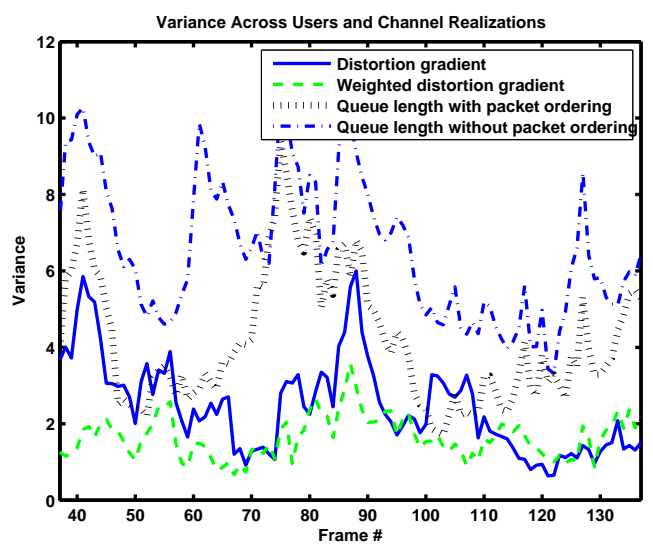

(b)

Fig. 2. Comparison of Resource Allocation Schemes using Simple Error Concealment. (a) Average PSNR per user. User numbers represent 1: Foreman, 2: Mother and Daughter, 3: Carphone, 4: News, 5: Silent, 6: Hall Monitor. (b) Variance across users and channel realizations

We performed simulations to determine the performance gain that can be expected by using the content-dependent packet ordering and resource allocation scheme. Lost packets were concealed using the simple concealment technique described above. Six video sequences with varied content: "foreman", "carphone", "mother and daughter", "news", "hall monitor", and "silent", in QCIF (176x144) format were used for the simulations. The sequences were encoded in H.264 (JVT reference software, JM 9.3 [30]) at variable bit rates to obtain a specified average PSNR of $35 \mathrm{~dB}$ for each frame. All frames except the first were encoded as $\mathrm{P}$ frames. To reduce error propagation due to packet losses, random I MBs were inserted into each frame during the encoding process. The frames were packetized such that each packet/slice contained one row of MBs, which enabled a good balance between error robustness and compression efficiency. Constrained intra prediction was used at the encoder for further error robustness. Although the sequences begin transmitting simultaneously, we provide a buffer of 10 frame times in order for the first frame (Intra coded) to be received by each user. Therefore, the start times of the subsequent frames can vary for each user. If a video packet could not be completely transmitted within a given transmission opportunity, we assume that it can be fragmented, and the utility gradient of the fragmented packet is calculated using the 
TABLE I

System Parameters USEd In Simulations

\begin{tabular}{|c|c|c|c|c|}
\hline$N$ & $N_{i}$ & $P$ & $\breve{s}_{i}$ & $s_{i}$ \\
\hline 15 & 5 & $10 \mathrm{~W}$ & 0 & $1.76 \mathrm{~dB}$ \\
\hline
\end{tabular}

number of remaining bits to be transmitted.

The wireless network was modeled as an HSDPA system. The system parameters used in the simulations are shown in Table I. HSDPA provides $2 \mathrm{msec}$ transmission time slots. Realistic channel traces for an HSDPA system were obtained using a proprietary channel simulator developed at Motorola Inc. The simulator accounts for correlated shadowing and multipath fading effects with 6 multipath components. For the channel traces, users were located within a $0.8 \mathrm{~km}$ radius from the base station and user speeds were set at $30 \mathrm{~km} / \mathrm{h}$. Figure 2 compares the average quality of the received video, using 4 different methods for calculating the utilities in (8). The first sets $w_{i}=1$ for all $i$ and uses the utility functions described in Sec. III. The second, is a modification of the first, where $w_{i}$ is set to be the distortion of the currently transmitted sequence of user $i$ to ensure fairness across users. The third method is only partially content-aware in that it orders the video packets of each user according to their importance. The resource allocation across users, however, is performed assuming that the utility gradients in (8) are proportional to the current queue length in bits of each user's transmission queue. The computational complexity of the first three methods is very similar as they all use the proposed packet ordering scheme. The final method is similar to the conventional content-independent scheduling techniques in which no packet ordering is performed at the scheduler; Scheduling is again based on queue sizes.

Figure 2(a) shows the average quality across 100 frames over 5 channel realizations for each sequence. This shows that the content-aware schemes significantly out-perform the conventional queue length based scheduling scheme. The gain in performance is mainly seen in the sequences with more complex video content across the entire frame such as foreman, mother and daughter, and carphone. The content aware schemes recognize the importance of error concealment in enabling packets in more easily concealable sequences such as news and hall monitor to be dropped, while the contentindependent schemes do not. Figure 2(b) shows the variance in PSNR per frame across all users and the 5 channel realizations. This shows that the two schemes with content-aware gradient metrics tend to provide similar quality across all the users (lower variance), while the queuedependent schemes tend to favor some users, again those whose dropped packets would have 
been easily concealable, over others. Between the two schemes with content-aware metrics, we can see that a small sacrifice in average PSNR incurred by the weighted distortion gradient metric yields significant improvement in terms of the variance across users.

2) Complex Error Concealment: A broad review of error concealment techniques can be found in [31], [32]. Error concealment exploits spatial and temporal redundancies in the video data. In complex temporal concealment techniques, the motion vectors (MV's) of neighboring decoded MB's in the frame are used to estimate the motion vector of a lost MB. For example, one possibility is to use the median MV of all the available neighboring MV's. Another is to use a boundary matching technique to determine the best candidate MV [33]. Errors in intra frames are concealed using spatial concealment techniques that rely on weighted pixel averaging schemes where the weight depends on the distance from the concealed pixels. More complex hybrid spatiotemporal error concealment techniques also exist [34].

When complex concealment is used, the packet ordering scheme proposed in Sec. III changes, and the incremental gain in quality due to adding each packet is no longer additive. Figure 3 illustrates this issue for a particular frame of the foreman sequence. In Fig. 3(a) the packet representing the $5^{\text {th }}$ row of MBs is the only packet received from the frame, and the rest of the MBs are concealed using that packet. In (b), the $6^{\text {th }}$ row is the only row received, and in (c), both the $5^{\text {th }}$ and $6^{\text {th }}$ rows are received. The darker pixels in each figure indicate higher gains in quality compared to not receiving any packets at all. We can see that, due to concealment, the effect of receiving one packet extends beyond the immediate region represented by the packet, and that therefore, adding the $6^{\text {th }}$ packet to the already transmitted $5^{\text {th }}$ packet does not provide an incrementally additive gain in quality corresponding to the gain that would occur if only the $6^{\text {th }}$ packet were received.

Our solution, formulated in (2) and (3), takes into account the non-additivity of packet utilities by employing a myopic method for determining the packet orderings within the transmission queue. For each position in the transmission queue, we choose the packet that provides the largest gain in quality after error concealment, given the packets that have already been added to the queue. Figure 4 shows an example user utility function obtained with the myopic packet ordering scheme. We can see that the error concealment causes the utility function to not be concave over the entire range. A result of this is that a packet may have lower priority, preventing a future packet with 


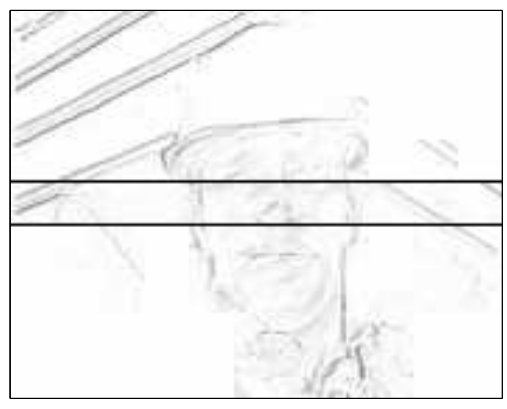

(a) MSE Gain $=262$

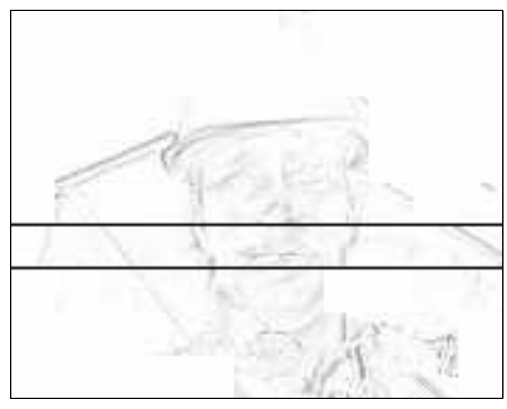

(b) MSE Gain $=137$

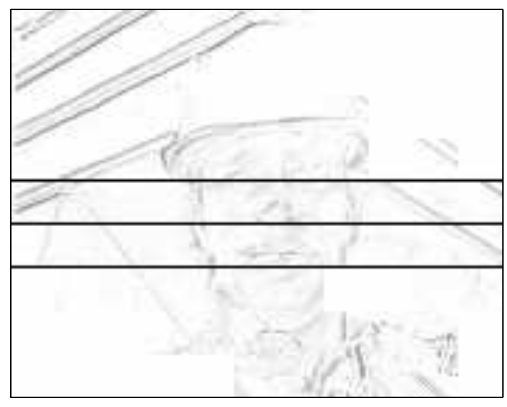

(c) MSE Gain $=272$

Fig. 3. Non-additive gain in quality due to complex concealment. Darker pixels indicate higher gain compared to not receiving any packets from the frame. The row borders are shown in black. (a) Packet containing MB row 5 received, (b) MB row 6 received, (c) MB rows 5 and 6 received (Total MSE gain signifi cantly less than the sum of (a) and (b))

higher value from being transmitted. To avoid this problem, when determining the utility gradients to be used in (8), we instead consider a smoothed utility gradient using,

$$
u_{i, \pi_{i, k_{i}+1}}\left[k_{i}\right]=\frac{D_{i}\left[\Pi_{i}\left(k_{i}\right)\right]-D_{i}\left[\Pi_{i}\left(k_{i}+L\right)\right]}{\sum_{m=k_{i}+1}^{k_{i}+L} b_{i, \pi_{i, m}}}
$$

where $L$ is a window of succeeding packets over which the gradient is calculated. In Fig. 5, we show simulation results using the same encoded sequences as in Sec. VI-A.1, and the same system parameters as in Table I, where the performance due to using simple and complex concealment techniques is compared. In calculating the smoothed utility gradients as in (18), we set $L=3$, which was empirically found to be an appropriate choice. The results are averaged over 100 frames and 5 channel

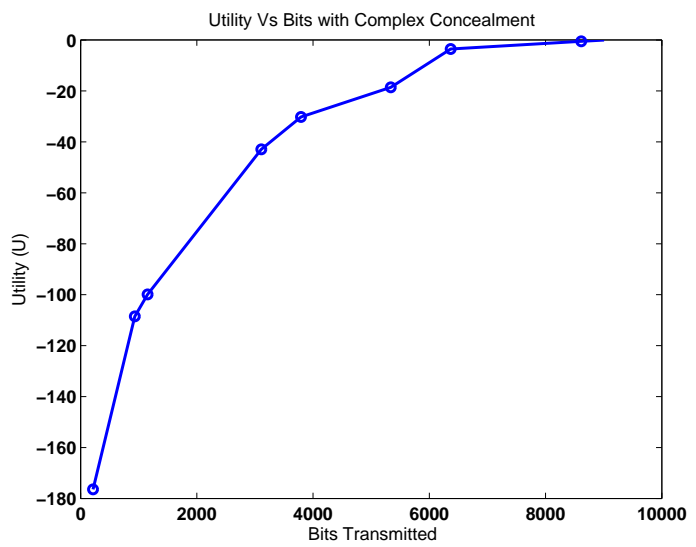

Fig. 4. User utility function after packet ordering with myopic technique for complex concealment. The markers indicate bit boundaries for each packet.

realizations. Here, we also consider the case where the decoder uses a complex concealment technique but at the scheduler, a simple concealment technique is assumed during the packet ordering and resource allocation process. When simple concealment is assumed, a 


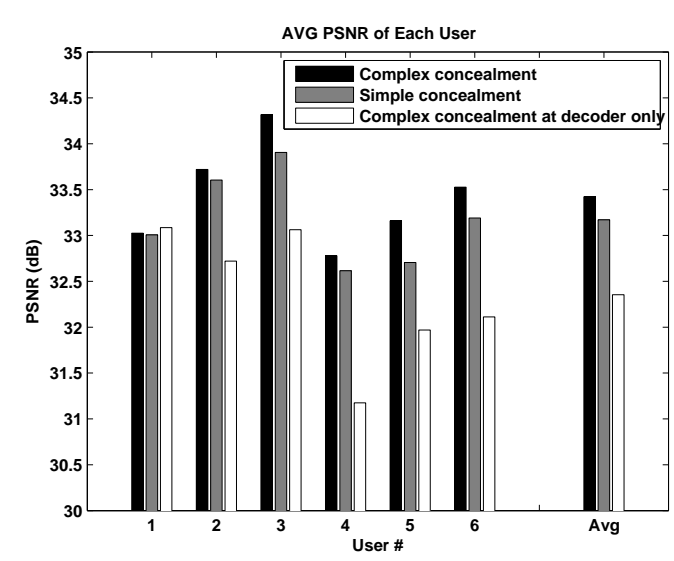

Fig. 5. Performance comparison using simple and complex error concealment techniques at the decoder.

video frame needs to be decoded only once in order to determine the utility gradients of each packet. When complex concealment is used, however, the video frame must be decoded $M$ times, where $M$ is the number of packets in the frame, to determine the concealment effect of each packet. From Fig. 5, we can see that, though the packet ordering scheme with complex concealment is suboptimal, the performance of the system improves overall, as well as for most of the individual sequences. Not taking into account the decoder error concealment technique at the scheduler leads to a significant degradation in performance.

\section{B. Offline or Simplified Packet Ordering Schemes}

As temporal concealment, whether simple, or complex, uses information from previously decoded frames, the described packet ordering techniques require knowledge of the decoder state up to the previously transmitted frame. The decoder state at any time, however, is dependent on the specific channel realization up to that time, as well as the congestion in the network. Therefore, to achieve best results, the packet ordering must be done in real-time at the scheduler, which implies that the scheduler must be able to decode the video sequence given a specified set of packets, and determine the quality of the decoded video, in real-time.

Assuming that not all schedulers will have the necessary computational power to order the packets in real-time, we have considered a suboptimal technique for determining the packet ordering offline. An application of the technique, termed "Offline1" in Fig. 6, is to assume that the decoder state up to the previous packet group is perfect (i.e. all previous packets are received without loss), when ordering the packets for the current group. A further extension of this method, termed "Offline2" is to assume that the decoder state up to all but the previous packet group is perfect, which assumes a firstorder dependency among packet groups. In these methods, each packet can be stamped offline at the media server with an identifier marking its order within the packet group, as well as a utility gradient, which can be directly used by the scheduler. In the case of "Offline2", 


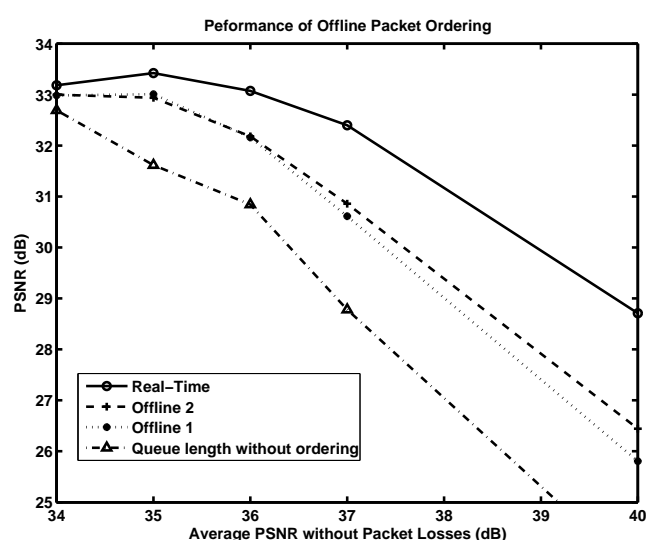

(a)

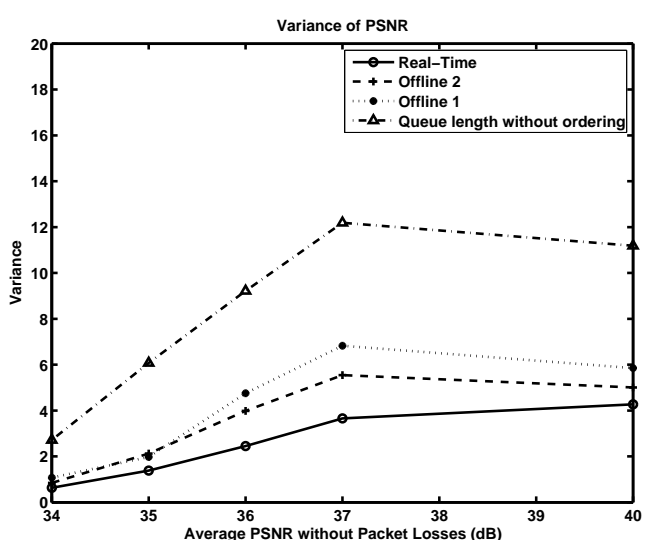

(b)

Fig. 6. Comparison of content-dependent offlne ordering methods with real-time ordering and content-independent queue length based scheme. (a) Average PSNR over all users and channel realizations. (b) Variance of PSNR across all users and channel realizations

each packet will need to be marked with $M$ different priority values where each value corresponds to the number of packets transmitted from the previous packet group. In Fig. 6, we plot the performance of each system, real-time, Offline1, and Offline2, as the quality of the initially encoded sequence is increased. We also compare these content dependent schemes to the previously discussed content-independent queue length based scheme without packet ordering. Again, the system parameters in Table I are used. Figure 6(a) shows the average PSNR over all users and channel realizations and Figure 6(b) shows the variance of PSNR across all users and channel realizations averaged over all frames of the sequence. As the initial qual- ity increases, the bit rates of the sequences increase, leading to higher packet losses. As the number of packet losses increases, the gap between the real-time and offline methods also increases. When the initial quality is $34 \mathrm{~dB}$ and $35 \mathrm{~dB}$, however, where the percentage of packets dropped per frame per user for the offline methods, is $10 \%$ and $16 \%$, respectively, we can see that the performance of the offline methods remains close to that of the real-time scheme. This suggests that, if the video encoding is well matched to the channel, the offline schemes perform well but when mismatch occurs, the performance degrades. The offline packet prioritization schemes, however, still perform significantly better than queue dependent scheduling 
without packet prioritization. We should note that, although it performs slightly better, the "Offline2" method does not show a significant gain over the more simple "Offline1" method.

\section{Error Resilient Video Encoding for Stream- ing Over Wireless Packet Access Networks}

When scheduling and transmitting preencoded video packets over wireless channels, some packets are inevitably dropped due to inadequate channel resources. Error resilient video encoding schemes alleviate the ill-effects of packet loss on the decoded video [35]. Error robust video compression, however, involves a trade-off with greater robustness leading to lower compression efficiency. Therefore, the performance of specific error resilience tools and compression schemes must be analyzed under realistic channel conditions. This section examines some of the trade-offs important to this work.

Among the tools that trade-off compression efficiency for error resilience are the slice structure, which allows for resynchronization within a frame, flexible macroblock ordering, which enables better error concealment, and constrained intra prediction as well as random I MB insertion, which reduce error propagation. In our numerical work, we have assumed a slice structure consisting of one row of MBs per slice, which achieves a reasonable compro- mise between error robustness and compression efficiency.

In table II, we show the trade-off between error resilience and compression efficiency due to random I MB insertion. The system parameters are kept the same as in the previous simulations and the performance results are shown for the Foreman sequence given that each of the six sequences is initially encoded using the given numbers of random I MBs per frame. The quality of the encoded sequence without packet losses is maintained close to $35 \mathrm{~dB}$ through rate control. We can see that as the number of random I MBs increases, the bit rate of the encoded stream increases, which leads to higher packet drop rates at the scheduler and resultant loss in video quality. Not using I MBs also degrades the video quality by increasing error propagation. Similarly, in Fig. 7, we show a comparison between sequences encoded using intra prediction, a technique proposed in H.264 to increase compression efficiency, and those encoded using constrained intra prediction. In intra prediction, intra MBs are predictively dependent on neighboring MBs, some of which may be inter, of the same slice. In a packet lossy system, such dependencies lead to error propagation. Constrained intra prediction limits intra prediction to using only the neighboring intra MBs, which eliminates error propagation at the cost of lower compression efficiency. 
TABLE II

TRADE-OFF BETWEEN ERROR RESILIENCE AND

COMPRESSION EFFICIENCY DUE TO RANDOM I MB

INSERTION

\begin{tabular}{|c|c|c|c|c|}
\hline $\begin{array}{c}\text { Random } \\
\text { I MBs }\end{array}$ & $\begin{array}{c}\text { Input Rate } \\
\text { (kbps) }\end{array}$ & $\begin{array}{c}\text { Pct Pkts } \\
\text { Dropped }\end{array}$ & $\begin{array}{c}\text { Received Avg } \\
\text { PSNR(dB) }\end{array}$ & $\begin{array}{c}\text { PSNR } \\
\text { Loss }\end{array}$ \\
\hline 0 & 153 & 1.0 & 32.7 & 2.8 \\
2 & 153 & 0.4 & 33.4 & 1.6 \\
$\mathbf{4}$ & $\mathbf{1 7 6}$ & $\mathbf{0 . 8}$ & $\mathbf{3 4 . 3}$ & $\mathbf{0 . 9}$ \\
6 & 200 & 1.6 & 34.1 & 1.4 \\
8 & 200 & 2.1 & 33.6 & 1.4 \\
10 & 200 & 2.8 & 33.2 & 1.4 \\
12 & 248 & 5.1 & 32.6 & 2.8 \\
\hline
\end{tabular}

From Fig. 7, it is apparent that the gain in compression efficiency due to intra prediction is not sufficient to offset the performance loss due to error propagation.

A relationship between the source encoding rate and the quality of the received video can also be determined. Given similar channel conditions, lower source rates lead to lower packet losses at the cost of higher distortion due to compression artifacts. On the other hand, higher source rates can lead to lower compression artifacts, at the expense of higher packet losses, some of which can be concealed. We use our channel simulations with varying source encoding rates to determine the optimal encoding rates under the given average channel

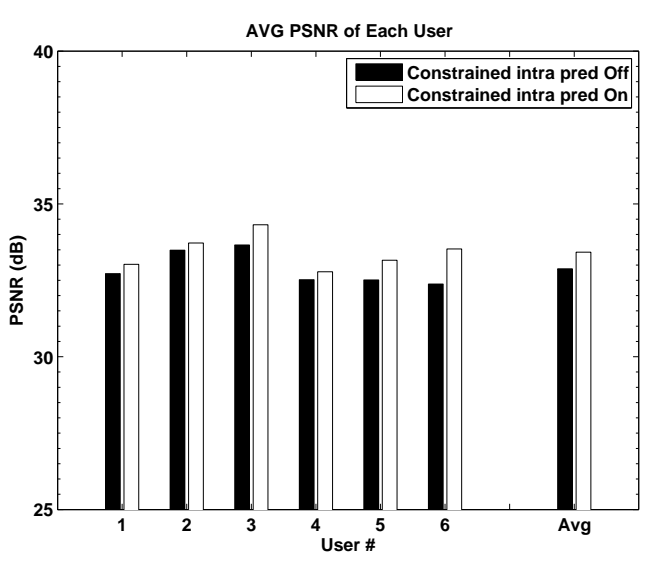

Fig. 7. PSNR of received video if original video is encoded with and without constrained intra prediction. Average quality without packet losses for all sequences is close to $35 \mathrm{~dB}$.

conditions. Figure 8 shows the performance results for a multiple user system where each user's sequence is initially encoded such that the decoded quality without packet losses is close to the specified average PSNR. Then, we measure the decoded PSNR after packets are dropped at the transmission queue using our packet scheduling scheme. Figure 8 shows that, given the average channel conditions, it is possible to find an appropriate source rate for the pre-encoded video sequences. Therefore, the media server could potentially keep multiple source bit streams at different rates for each video sequence and choose the appropriate stream based on the average channel conditions. 


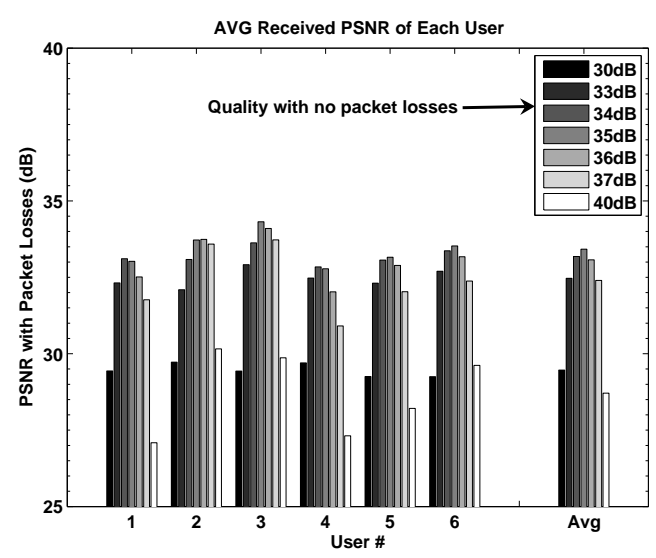

Fig. 8. PSNR of received video with varying initial bit rates corresponding to varying quality prior to transmission losses.

\section{CONCLUSIONS}

We have shown that a resource allocation scheme that maximizes a weighted sum of the rates assigned to each user where the weights are determined by distortion-based utility gradients, is a simple but effective solution for downlink packet scheduling in wireless video streaming applications. We have provided an optimal solution for the case when the video packets are independently decodable and a simple error concealment scheme is used at the decoder. We have also shown that with complex error concealment at the decoder, a suboptimal myopic solution with appropriately calculated distortion utility gradients can still provide excellent results. We show the dependency of the system on the compression and error resilience schemes used at the encoder. In the future we plan to do a more comprehensive study of the performance tradeoffs that may be obtained by intelligently coding the input bit streams. Another future direction is to explore the use of scalable video coding algorithms, which allow for spatial, temporal, and quality scalability within a single bit stream. Our technique can easily be adapted to temporal and quality scalable bit streams, given a reasonable metric is used to measure the distortion due to lost or partially transmitted video packets.

In conclusion, we would like to thank Rajeev Agrawal and Hua $\mathrm{Xu}$, at Motorola Inc, Arlington Heights, IL, for their valuable advice and support.

\section{REFERENCES}

[1] High Speed Downlink Packet Access; Overall Description. 3GPP Std. TS 25.308 v7.0.0, 2006.

[2] IEEE Standard for Local and Metropolitan Area Networks; Part 16: Air Interface for Fixed Broadband Wireless Access Systems. IEEE Std 802.16e, 2005.

[3] S. Lu, V. Bharghavan, and R. Srikant, 'Fair Scheduling in Wireless Packet Networks," ACM SIGCOMM Computer Communication Review, vol. 27, no. 3, October 1997.

[4] R. Agrawal, A. Bedekar, R. La, and V. Subramanian, "A Class and Channel-Condition based Weighted Proportionally Fair Scheduler," in Proc. of ITC, Sep 2001.

[5] S. Shakkottai, R. Srikant, and A. Stolyar, 'Pathwise Optimality and State Space Collapse for the Exponential Rule," in Proc. of the IEEE International Symposium on Information Theory, 2002.

[6] Y. Liu and E. Knightly, 'Opportunistic Fair Scheduling over Multiple Wireless Networks," in Proc. of IEEE INFOCOMM, March 2003. 
[7] P. Liu, R. Berry, and M. Honig, 'Delay Sensitive Packet Scheduling in Wireless Networks," in Proc. of IEEE WCNC 2003, March 2003.

[8] R. Knopp and P. Humblet, 'Information Capacity and Power Control in Single-Cell Multiuser Communications," in Proc. of IEEE Int. Conference on Communications, 1995.

[9] D. Tse, "Optimal Power Allocation over Parallel Gaussian Broadcast Channels," in Proc. of ISIT, 1997.

[10] L. Li and A. Goldsmith, 'Optimal Resource Allocation for Fading Broadcast Channels- Part I: Ergodic Capacity," IEEE Trans. Information Theory, March 2001.

[11] R. Agrawal and V. Subramanian, 'Optimality of Certain Channel Aware Scheduling Policies," in Proc. of 2002 Allerton Conf. on Communication, Control and Computing, October 2002.

[12] R. Agrawal, V. Subramanian, and R. Berry, 'Joint Scheduling and Resource Allocation in CDMA Systems," IEEE Trans. Information Theory, to appear.

[13] R. O. Hinds, 'Robust mode selection for block-motioncompensated video encoding," Ph.D. dissertation, MIT, Cambridge, MA, 1999.

[14] R. Zhang, S. L. Regunathan, and K. Rose, "Video encoding with optimal Inter/Intra-mode switching for packet loss resilience," IEEE Journal on Selected Areas in Communications, vol. 18, pp. 966-976, June 2000.

[15] Z. He, J. Cai, and C. W. Chen, 'Joint source channel ratedistortion analysis for adaptive mode selection and rate control in wireless video coding," IEEE Trans. Circuits and Systems for Video Technology, vol. 12, no. 6, pp. 511-523, June 2002.

[16] C. E. Luna, Y. Eisenberg, R. Berry, T. N. Pappas, and A. K. Katsaggelos, 'Joint source coding and data rate adaptation for energy effi cient wirless video streaming," IEEE Journal on Selected Areas in Communications, vol. 21, no. 10, pp. 1710-1720, December 2003.

[17] Y. Eisenberg, C. E. Luna, T. N. Pappas, R. Berry, and A. K. Katsaggelos, 'Joint source coding and transmission power management for energy effi cient wireless video communications," IEEE Trans. Circuits and Systems for
Video Technology, vol. 12, no. 6, pp. 411-424, June 2002.

[18] Y. Eisenberg, F. Zhai, T. N. Pappas, R. Berry, and A. K. Katsaggelos, "VAPOR: variance-aware per-pixel optimal resource allocation," IEEE Trans. Image Processing, vol. 15, no. 2, pp. 289-299, February 2006.

[19] A. Katsaggelos, Y. Eisenberg, F. Zhai, R. Berry, and T. Pappas, "Advances in Effi cient Resource Allocation for Packet-Based Real-Time Video Transmission," Proc. IEEE, vol. 93, no. 1, pp. 135-147, January 2005.

[20] P. Chou and Z. Miao, 'Rate Distortion Optimized Streaming of Packetized Media," IEEE Trans. Multimedia, vol. 8, no. 2, pp. 390-404, April 2006.

[21] J. Chakareski, P. Chou, and B. Aazhang, 'Computing Rate-Distortion Optimized Policies for Streaming Media to Wireless Clients," in Proc. IEEE Data Compression Conference, April 2002.

[22] Z. Miao and A. Ortega, "Optimal Scheduling for Streaming of Scalable Media," in Proc. of Asilomar, November 2000.

[23] Y. Ofuji, S. Abeta, and M. Sawahashi, 'Unifi ed Packet Scheduling Method Considering Delay Requirement in Forward Link Broadband Wireless Access," in Proc. of Vehicular Technology Conference, Fall 2003.

[24] P. Falconio and P. Dini, 'Design and Performance Evaluation of Packet Scheduler Algorithms for Video Traffi c in the High Speed Downlink Packet Access," in Proc. of PIMRC 2004, September 2004.

[25] D. Kim, B. Ryu, and C. Kang, 'Packet Scheduling Scheme for real time Video Traffic in WCDMA Downlink," in Proc. of 7th CDMA International Conference, October 2002.

[26] R. Tupelly, J. Zhang, and E. Chong, 'Opportunistic Scheduling for Streaming Video in Wireless Networks," in Proc. of Conference on Information Sciences and Systems, 2003.

[27] G. Liebl, T. Stockhammer, C. Buchner, and A. Klein, 'Radio Link Buffer Management and Scheduling for Video Streaming Over Wireless Shared Channels," in Proc. of the Packet Video Workshop, 2004.

[28] G. Liebl, M. Kalman, and B. Girod, 'Deadline-Aware 
Scheduling for Wireless Video Streaming," in Proc. IEEE Int. Conf. on Multimedia and Expo, July 2005.

[29] J. Huang, V. Subramanian, R. Agrawal, and R. Berry, 'Downlink Scheduling and Resource Allocation for OFDM Systems," in Conference on Information Sciences and Systems (CISS 2006), March 2006.

[30] JVT Reference Software. http://iphome.hhi.de/suehring/ $\mathrm{tml} /$ download, JM 9.3.

[31] M. Hong, L. Kondi, H. Schwab, and A. Katsaggelos, 'Error Concealment Algorithms for Concealed Video," Signal Processing: Image Communications, special issue on Error Resilient Video, vol. 14, no. 6-8, pp. 437-492, 1999.

[32] Y. Wang and Q.-F. Zhu, 'Error control and concealment for video communication: a review," Proc. IEEE, vol. 86, pp. 974-997, May 1998.

[33] Y.-K. Wang, M. Hannuksela, V. Varsa, A. Hourunranta, and M. Gabbouj, "The Error Concealment Feature in the H.26L Test Model," in Proc. IEEE Int. Conference on Image Processing, vol. 2, September 2002, pp. 729-732.

[34] S. Belfi ore, M. Grangetto, E. Magli, and G. Olmo, 'Spatio-Temporal Video Error Concealment with Perceptually Optimized Mode Selection," in Proc. IEEE Int. Conf. Acoust., Speech and Signal Processing, vol. 5, 2003, pp. 748-751.

[35] Y. Wang, G. Wen, S. Wenger, and A. Katsaggelos, 'Review of Error Resilient Techniques for Video Communications," IEEE Signal Processing Magazine, vol. 17, no. 4, pp. 61-82, July 2000.

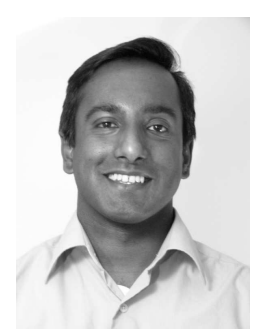

Peshala V. Pahalawatta received the B.S. degree in Electrical Engineering from Lafayette College, Easton, Pennsylvania, in 2000, and the M.S. degree in Electrical and Computer Engineering from Northwestern University, Evanston, Illinois, in 2002. He is currently pursuing a Ph.D. degree in Electrical and Computer Engineering at Northwestern University. His primary research interests include image and video compression and transmission, wireless communication, and computer vision.

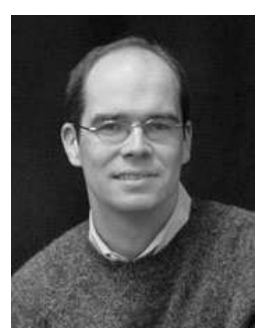

Randall A. Berry received the B.S. degree in Electrical Engineering from the University of Missouri-Rolla in 1993 and the M.S. and $\mathrm{PhD}$ degrees in Electrical Engineering and Computer Science from the Massachusetts Institute of Technology in 1996 and 2000, respectively. In September 2000, he joined the faculty of Northwestern University, where he is currently an Associate Professor in the Department of Electrical Engineering and Computer Science. In 1998 he was on the technical staff at MIT Lincoln Laboratory in the Advanced Networks Group, where he worked on optical network protocols. His current research interests include wireless communication, data networks and information theory.

Dr. Berry is the recipient of a 2003 NSF CAREER award and the 2001-02 best teacher award from the ECE Department at Northwestern. He is currently serving on the editorial board of IEEE Transactions on Wireless Communications and is a guest editor of an upcoming special issue of IEEE Transactions on Information Theory on 'Relaying and Cooperation in Networks." 


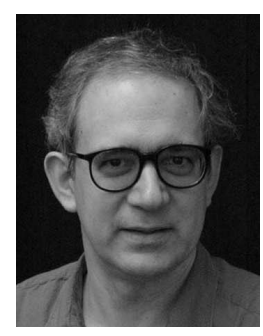

Thrasyvoulos N. Pappas received the S.B., S.M., and Ph.D. degrees in Electrical Engineering and Computer Science from the Massachusetts Institute of Technology, Cambridge, MA, in 1979, 1982, and 1987, respectively. From 1987 until 1999, he was a Member of the Technical Staff at Bell Laboratories, Murray Hill, NJ. In September 1999, he joined the Department of Electrical and Computer Engineering at Northwestern University as an associate professor. His research interests are in image and video compression, video transmission over packet-switched networks, perceptual models for image processing, model-based halftoning, image and video analysis, video processing for sensor networks, audiovisual signal processing, and DNA-based digital signal processing.

Dr. Pappas is a Fellow of the IEEE. He has served as chair of the IEEE Image and Multidimensional Signal Processing Technical Committee, associate editor and electronic abstracts editor of the IEEE Transactions on Image Processing, technical program co-chair of ICIP-01 and the Symposium on Information Processing in Sensor Networks (IPSN-04), and since 1997 he has been co-chair of the SPIE/IS\&T Conference on Human Vision and Electronic Imaging. He was also co-chair for the 2005 IS\&T/SPIE Symposium on Electronic Imaging: Science and Technology.

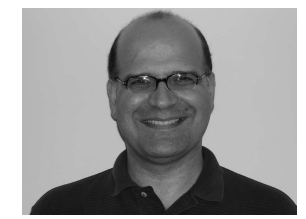

Aggelos K. Katsaggelos received the Diploma degree in Electrical and Mechanical Engineering from the Aristotelian University of Thessaloniki, Greece, in 1979 and the M.S. and Ph.D. degrees both in electrical engineering from the Georgia Institute of Technology, in 1981 and 1985, respectively. In 1985 he joined the Department of Electrical Engineering and Computer Science at Northwestern University, where he is currently professor. He is also the Director of the Motorola Center for Seamless Communications and a member of the Academic Affi liate Staff, Department of Medicine, at Evanston Hospital.

Dr. Katsaggelos is a member of the Publication Board of the IEEE Proceedings, the IEEE Technical Committees on Visual Signal Processing and Communications, and Multimedia Signal Processing, the Editorial Board of Academic Press, Marcel Dekker: Signal Processing Series, Applied Signal Processing, and Computer Journal. He has served as editor-in-chief of the IEEE Signal Processing Magazine (1997-2002), a member of the Publication Boards of the IEEE Signal Processing Society, the IEEE TAB Magazine Committee, an Associate editor for the IEEE Transactions on Signal Processing (19901992), an area editor for the journal Graphical Models and Image Processing (1992-1995), a member of the Steering Committees of the IEEE Transactions on Image Processing (1992-1997) and the IEEE Transactions on Medical Imaging (1990-1999), a member of the IEEE Technical Committee on Image and Multi-Dimensional Signal Processing (1992-1998), and a member of the Board of Governors of the IEEE Signal Processing Society (1999-2001). He is the editor of Digital Image Restoration (Springer-Verlag 1991), co-author of RateDistortion Based Video Compression (Kluwer 1997), co-editor of Recovery Techniques for Image and Video Compression and Transmission, (Kluwer 1998), and co-author of SuperResolution for Images and Video, (Claypool, 2006). He was the holder of the Ameritech Chair of Information Technology (1997-2003), and he is the co-inventor of twelve international patents, a Fellow of the IEEE, and the recipient of the IEEE Third Millennium Medal (2000), the IEEE Signal Processing 
Society Meritorious Service Award (2001), an IEEE Signal

Processing Society Best Paper Award (2001), and an IEEE ICME Best Paper Award (2006). 